CONF. $970728--1$

\title{
SOLVING INVERSE PROBLEMS OF IDENTIFICATION TYPE BY OPTIMAL CONTROL METHODS*
}

\author{
${ }^{\oplus \cdot S}$. Lenhart, ${ }^{\circledR}$ V. Protopopescu, and ${ }^{\mp} \mathrm{J}$. Yong
}

\author{
"University of Tennessee \\ 'Center for Engineering Systems Advanced Research \\ Oak Ridge National Laboratory

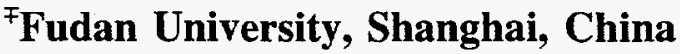

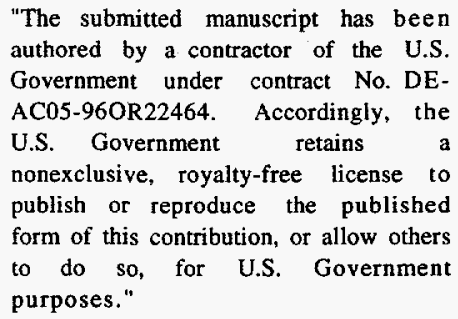

Submitted to: Int. Conf. on Applied Nonlinear Dynamics Near the Millenium, San Diego

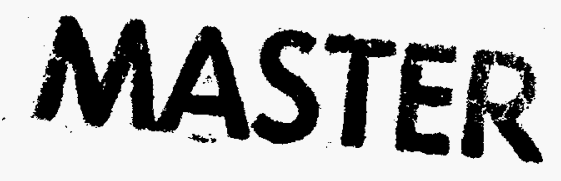

DISTRIBUTION OF THIS DOCUMENT IS UNLIMITED

\footnotetext{
* Research sponsored by the Engineering Research Program, Office of Basic Energy Sciences, of the U.S. Department of Energy, under contract DE-AC05-960R22464 with Lockheed Martin Energy Research, Inc.
} 


\section{DISCLAIMER}

This report was prepared as an account of work sponsored by an agency of the United States Government. Neither the United States Government nor any agency thereof, nor any of their employees, make any warranty, express or implied, or assumes any legal liability or responsibility for the accuracy, completeness, or usefulness of any information, apparatus, product, or process disclosed, or represents that its use would not infringe privately owned rights. Reference herein to any specific commercial product, process, or service by trade name, trademark, manufacturer, or otherwise does not necessarily constitute or imply its endorsement, recommendation, or favoring by the United States Government or any agency thereof. The views and opinions of authors expressed herein do not necessarily state or reflect those of the United States Government or any agency thereof. 


\section{DISCLAMMER}

Portions of this document may be illegible in electronic image products. Images are produced from the best available original document. 


\title{
Solving inverse problems of identification type by optimal control methods
}

\author{
Suzanne Lenhart \\ Mathematics Department, University of Tennessee, Knoxville, TN, 37996-1300 \\ Vladimir Protopopescu \\ Oak Ridge National Laboratory, Oak Ridge, TN, 37831-6364 \\ Jiongmin Yong \\ Mathematics Department, Fudan University, Shanghai, 200433, China
}

\begin{abstract}
Inverse problems of identification type for nonlinear equations are considered within the framework of optimal control theory. The rigorous solution of any particular problem depends on the functional setting, type of equation, and unknown quantity (or quantities) to be determined. Here we present only the general articulations of the formalism. Compared to classical regularization methods (e.g. Tikhonov coupled with optimization schemes), our approach presents several advantages, namely: (i) a systematic procedure to solve inverse problems of identification type; (ii) an explicit expression for the approximations of the solution; and (iii) a convenient numerical solution of these approximations.
\end{abstract}

\section{DIRECT PROBLEM}

We consider a system described by the state function $u$ which satisfies the abstract operator equation:

$$
A(u(\xi) ; F(\xi))=0 .
$$

The state of the system, $u$, and the data, $F$, can be scalar or vector and the operator $A$ is, in general, nonlinear. The independent variables of the problem, $\xi$, which in the evolution problem contain the time, $t$, take values in the domain $\Omega \subset \mathbb{R}^{n}$, with sufficiently regular boundary, $\partial \Omega$.

In order to fix ideas, we assume that the operator $A$ is a second order partial differential operator, but the present formalism is much more general, including equations of higher order, ordinary differential equations, hybrid systems, etc. The formulation applies as well to elliptic systems ( $u$ being independent of time) and hyperbolic (one redefines the state functions and rewrite the equation as a first order evolution system).

If the system (1) accurately describes a realistic physical situation, the direct problem is wellposed, i.e. the data $F$ (parameters, functions, coefficients, sources, initial and boundary values, etc.) determine uniquely and regularly the solution $u .^{1-3}$

\section{INVERSE PROBLEM}

In contrast, the inverse problem which consists of determining a part of $F$ from the solution $u$, is in general - ill-posed. ${ }^{4}$ In fact, in almost all the practical situations, the solution is never completely known and one must deal with only partial or imprecise knowledge of it. ${ }^{5}$ 
Formally, the inverse problem corresponding to the direct problem (1) is given below:

"Given partial (and perhaps noisy) observations, $B \tilde{u}$, of the true solution $\tilde{u}$, in a subdomain $\Omega^{\prime}$ of the phase space, $\Omega^{\prime} \subset \Omega$, and a known part, $f_{1}$, of the data $F$, we seek to determine the unknown part of the data, $f$."

This type of inverse problem corresponds to parameter identification in a model with known structure involving some unknown data. ${ }^{6}$ Other inverse problems corresponding to: (i) model identification, (ii) reconstructions from projections, or (iii) integral equations of the first type, etc. are not included in our approach.

The standard methods of solving these identification problems is based on Tikhonoff's regularization. In this approach, one constructs - starting from actual observations, $B \tilde{u}$, - a cost function:

$$
J_{\beta}(f)=\frac{1}{2}\|B u-B \tilde{u}\|^{2}+\frac{\beta}{2}\|f\|^{2}, \quad \beta>0
$$

where $u$ is the solution of (1) for the data $F=\left(f_{1}, f\right)$. The exact form of the cost functional and the types of norms involved depend on the concrete problem that has to be solved. The quadratic form used here makes the illustration clearer and simpler. Tikhonoff's approach and its variants seek to minimize the functional $J_{\beta}(f)$ - for a fixed $\beta$-over the set of unknown data. It assures - in principle - that the observation of the solution obtained with $f, B u$, will approach the actual observation, $B \tilde{u}$. The minimization is carried out by using an optimization program. The functions $f_{\beta}$ which achieve the absolute minimization of $J_{\beta}(f)$ represent the approximate solution of the inverse problem (2). The disadvantages of this approach are:

- when $\beta$ is very small, the problem is unstable;

- when $\beta$ is very large, the solution is not accurate;

- there is no systematic procedure for finding the absolute minimum;

- there are no systematic means to control the approximations;

- the optimization program is implemented by "trial and error" which makes the numerical calculations difficult and inefficient.

\section{SOLUTION OF THE INVERSE PROBLEM BY OPTIMAL CONTROL METHODS}

To eliminate most of the disadvantages above, we propose a new approach to the inverse problem of identification, based on optimal control for operator equations as developed by J.-L. Lions. ${ }^{7-9}$

Our idea is to consider a family of functionals (3) for $\beta \geq 0$. For each $\beta$ strictly positive one consides the unknownn data, $f$, as a control which belongs to a certain bounded set, $\mathcal{F}$; the control has to be adjusted - always remaining in the set - in order to minimize the functional $J_{\beta}(f)$. The minimum of the cost functional over $\mathrm{f}$ is attained at the optimal control, $f=f_{\beta}$ :

$$
J_{\beta}\left(f_{\beta}\right)=\inf _{f \in \mathcal{F}} J_{\beta}(f)
$$

Letting the sequence of $\beta$ tend toward zero, one can verify that the sequence $f_{\beta}$ converge in an appropriate sense to an element of the control set, $f^{*} \in \mathcal{F}$ (see Section 5 ). This element represents the solution - perhaps non-unique - of the inverse problem (2). 
The idea to use the techniques of optimal control to approximate in a systematic manner the solution of an inverse identification problem is new. Indeed, as mentioned above, the majority of the traditional approaches ${ }^{4-6}$ couple Tikhonoff's regularization with an optimization algorithm. Recently, Puel et Yamamoto ${ }^{10,11}$ have obtained uniqueness and stability results and reconstruction algorithms for the identification of sources in a wave equation. Their work is based on the results of exact controllability and uniqueness of the Hilbert uniqueness method (HUM) obtained by J.-L. Lions ${ }^{12-14}$. These results are limited to linear problems and moreover the identication is realized for a special type of observations. Finally, the other approaches which use the control theory - not necessarily optimal - for solving inverse problesm are limited to a particular cases where: (i) the equation is specified ${ }^{15}$; (ii) the control problem is the linear quadratic type and can be treated by Riccati equations ${ }^{16}$; or (iii) there is a bilinear control of a concrete type ${ }^{17,18}$.

\section{SOLUTION OF OPTIMAL CONTROL PROBLEM}

To prove that there exists an optimal control $f_{\beta}$ which minimizes $(3)$ one first establishes the needed existence, uniqueness, and a priori estimates for the solution $u$ of the direct problem (1) and uses a minimizing sequences argument. To characterize the optimal control, one procedes as follows:

- One shows that the function $f \rightarrow u(f)$ is differentiable. In general, there exists a directional derivative (evaluated at the optimal control, $f_{\beta}$ ):

$$
\left.\frac{\partial u}{\partial f}\right|_{f_{\beta}, \text { direction } \ell}=\psi
$$

which is the solution of the linear problem:

$$
L\left(\psi ; f_{1}, f_{\beta}, \ell, u_{\beta}\right)=0
$$

corresponding to problem (1). The operator $L$ is linear in $\psi$ and depends nonlinearly on $f_{\beta}$ and on $u_{\beta}=u\left(f_{\beta}\right)$.

- By differentiating $J_{\beta}(f)$ and using the estimates on $u$ and $\psi$, one obtains the characterization of the optimal control $f_{\beta} \in \mathcal{F}$ that minimizes $J_{\beta}$ over $\mathcal{F}$.

- In deriving this characterization, one constructs the adjoint system corresponding to (1), for the adjoint function $p$ :

$$
L^{*}\left(p ; f_{1}, f_{\beta}, u_{\beta}\right)=0
$$

and the resulting characterization for $f_{\beta}$ in terms of state and adjoint variables:

$$
f_{\beta}=G\left(u_{\beta}, p\right) \text {. }
$$

- Replacing $f_{\beta}$ in (1) and (7) by the characterization (8), one obtains the optimality system (OS):

$$
\begin{aligned}
A\left(u_{\beta} ; f_{1}, G\left(u_{\beta}, p\right)\right) & =0 \\
L^{*}\left(p ; f_{1}, G\left(u_{\beta}, p\right), u_{\beta}\right) & =0 .
\end{aligned}
$$

- The uniqueness of the solution of the OS guarantees the uniqueness of the optimal control, but - in general - the OS admits more than one solution, and then one has to analyze the uniqueness of $f_{\beta}$ by other methods. 
- Finally, the solution (the solutions) of the OS are calculated numerically by applying an iterative algorithm for two point boundary value problem (TPBVP).

\section{PASSAGE TO THE LIMIT AND SOLUTION OF THE INVERSE PROBLEM}

The cost functional (3) is an approximation in the sense that the second term is artificial - as always in Tikhonoff regularization. By letting $\beta$ tend toward zero one expects to obtain a control $f_{0}$ which minimizes only the difference between the actual observation and the solution of (1) with $f$ replaced by $f_{0}$.

We assume that the inverse problem (2) has a solution, i.e. there exists $f^{*} \in \mathcal{F}$ such that $u^{*}=u\left(f^{*}\right)$ satisfies $B u^{*}=B \tilde{u}$ a.e. in $\Omega^{\prime}$. Then we can prove that there exists $f_{0}$ (not necessarily equal to $f^{*}$ such that on a (sub)sequence $\beta \rightarrow 0$, we have

$$
\begin{aligned}
& f_{\beta} \rightarrow f_{0} \\
& u_{\beta}=u\left(f_{\beta}\right) \rightarrow u_{0}
\end{aligned}
$$

and

$$
B u_{0}=B \tilde{u} \quad \text { a.e. in } \Omega^{\prime} \text {. }
$$

The generic idea of justifying the result is the following:

With $f^{*} \in \mathcal{F}$, one has $J_{\beta}\left(f_{\beta}\right) \leq J_{\beta}\left(f^{*}\right)$, i.e.,

$$
\frac{1}{2}\left\|B u_{\beta}-B \tilde{u}\right\|^{2}+\frac{\beta}{2}\left\|f_{\beta}\right\|^{2} \leq \frac{\beta}{2}\left\|f^{*}\right\|^{2} .
$$

By utilizing the a priori estimates independent of $\beta$ obtained on $u_{\beta}$ one has on a subsequence of $\beta \rightarrow 0$, that $f_{\beta}$ goes toward $f_{0}$ and $u_{\beta}$ goes toward $u_{0}$ (with convergence of appropriate derivatives); moreover, $u_{0}=u\left(f_{0}\right)$. Letting $\beta$ tend to 0 in (11), one also obtains $B u_{0}=B \tilde{u}$. Thus, in the observation domain the solution $u_{0}$ is close (ideally identical) to the actual measured values and so the control $f_{0}$ is identified.

Remarks. The condition required to ensure (10) is equivalent to the observability condition used in HUM. This result is formal in the sense that the topologies of the limits are not specified.

\section{TWO EXAMPLES}

We applied the approach described above to solve two concrete identification problems motivated by underwater, geophysical, and seismic explorations.

6.1 Identification of the reflexion coefficient on a part of the boundary. ${ }^{17}$ For $\Gamma \subset \mathbb{R}^{2}, T>0$, and $w \in C(\Gamma), w<0$, we consider the spatio-temporal domain $\Omega=D \times(0, T)$, where $D=$ $\{(x, y, z) \mid(x, y) \in \Gamma, w(x, y)<z<0\}$. We divide the boundary of the domain $D$ in three disjoint parts:

$$
\begin{aligned}
& (\partial D)_{1}=\{(x, y, z) \mid(x, y) \in \partial \Gamma, w(x, y)<z<0\} \\
& (\partial D)_{2}=\Gamma \times\{z=0\} \\
& (\partial D)_{3}=\{(x, y, w(x, y)) \mid(x, y) \in \Gamma\}
\end{aligned}
$$


and consider the acoustic wave equation:

$$
\begin{array}{ll}
u_{t t}-\nabla(E \nabla u)=f_{1}, & \text { in } \Omega \\
u=0 & \text { on }(\partial D)_{1} \times(0, T) \\
\frac{\partial u}{\partial \nu}=0 & \text { on }(\partial D)_{2} \times(0, T) \\
\frac{\partial u}{\partial \nu}+f u=0 & \text { on }(\partial D)_{3} \times(0, T) \\
u=g_{1}, u_{t}=g_{2} & \text { in } D \times\{0\} .
\end{array}
$$

From observations $\tilde{u}$ of the solution of the system (12) effected in a subdomain $\Omega^{\prime} \subset \Omega$ we try to identify the reflection coefficient $f$ in a boundary condition of Robin type. As before, we consider that the control set is bounded, $0 \leq f \leq M$, and we look for a solution in $L^{2}\left(0, T ; H^{1}(\Omega)\right)$; the approximation $f_{\beta}$ minimizes

$$
J_{\beta}(f)=\frac{1}{2} \int_{\Omega^{\prime}}(u-\tilde{u})^{2}+\frac{\beta}{2} \int_{\Gamma} f^{2} .
$$

We obtain the explicit characterization

$$
f_{\beta}(x, y)=\min \left\{\left(\frac{1}{\beta} \int_{0}^{T} u_{\beta} p(x, y, w(x, y), t) d t\right)^{+}, M\right\}
$$

where the superscript + denotes the positive part of the function and $u_{\beta}$ is the solution of (12) for $f=f_{\beta}$ and $p$ is the solution of the adjoint system:

$$
\begin{array}{ll}
p_{t t}-\nabla(E \nabla p)=\left(u_{\beta_{z}}-\tilde{u}\right) \chi_{\Omega^{\prime}} & \text { in } \Omega \\
p=0 & \text { on }(\partial D)_{1} \times(0, T) \\
\frac{\partial p}{\partial \nu}=0 & \text { on }(\partial D)_{2} \times(0, T) \\
\frac{\partial p}{\partial \nu}+f u=0 & \text { on }(\partial D)_{3} \times(0, T) \\
p=p_{t}=0 & \text { in } D \times\{T\} .
\end{array}
$$

6.2 Identification of the potential for the wave equation. ${ }^{18}$ We describe the propagation of the acoustic wave equations in a spatial domain $D \subset \mathbb{R}^{3}$ by the system

$$
\begin{array}{ll}
u_{t t}=\Delta u+f u+f_{1} & \text { in } \Omega=D \times(0, T) \\
u=0 & \text { on } \partial D \times(0, T) \\
u=g_{1}, u_{t}=g_{2} & \text { in } D \times\{0\} .
\end{array}
$$

¿From observations $\tilde{u}$ effected in $\Omega^{\prime} \subset \Omega$ we try to determine the potential $f$. The control set is bounded, $|f| \leq M$, and we look for a solution in $L^{2}\left(0, T ; H_{0}^{1}(\Omega)\right)$; the approximation $f_{\beta}$ minimizes

$$
J_{\beta}(f)=\frac{1}{2} \int_{\Omega^{\prime}}(u-\tilde{u})^{2}+\frac{\beta}{2} \int_{\Omega} f^{2}
$$

We obtain the characterization

$$
f_{\beta}=\max \left(-M, \min \left(\frac{-u_{\beta} p}{\beta}, M\right)\right)
$$


where $u_{\beta}$ is the solution of the system (15) for $f=f_{\beta}$ and $p$ solves the adjoint problem:

$$
\begin{array}{ll}
p_{t t}-\Delta p=f_{\beta} p-\left(u_{\beta}-\tilde{u}\right) \chi_{\Omega^{\prime}} & \text { in } \Omega \\
p=0 & \text { on } \partial D \times(0, T) \\
p=p_{t}=0 & \text { in } D \times\{T\} .
\end{array}
$$

Remark. Both examples contain bilinear control problems.

\section{ACKNOWLEDGMENTS}

S.L. and V.P. acknowledge partial support from DOE'S Office of BES under contact No. AC0596OR22464 with Lockheed Martin Energy Research Corporation. J.Y. is supported in part by the NNSF of China, the Chinese State Education Commission Science Foundation, and the TransCentury Training Programme Foundation for Talents of the State Education Commission of China.

${ }^{1}$ J.-L. Lions, Équations Différentielles Opératorielles et Problèmes aux Limites, (Springer Verlag, Berlin, 1961).

${ }^{2}$ J.-L. Lions and E. Magenes, Non-Homogeneous Boundary Value Problems, vols. 1-3, (Springer Verlag, New-York, 1972).

${ }^{3}$ L. C. Evans, Partial Differential Equations, vols. 3A-3B (Berkeley Mathematics Lecture Notes, Berkeley, 1993).

${ }^{4} \mathrm{C}$. W. Groetsch, Inverse Problems in the Mathematical Sciences, (Vieweg, Braunschweig, Wiesbaden, 1993).

${ }^{5}$ I. J. D. Craig and C. Brown, Inverse Problems in Astronomy, (Adam Hilger Ltd., Bristol et Boston, 1986).

${ }^{6}$ H. T. Banks et K. Kunish, Estimation Techniques for Distributed Parameter Systems, (Birkhäuser, Boston, 1989).

${ }^{7}$ J.-L. Lions, Optimal Control of Systems Governed by Partial Differential Equations, (Springer Verlag, New York, 1971).

${ }^{8}$ J.-L. Lions, Some Methods in the Mathematical Analysis of Systems and Their Control, (Science Press, Beijing \& Gordon and Breach, New York, 1981).

${ }^{9}$ J.-L. Lions, Controllabilité Exacte, vols. 1-2, (Masson, Paris, 1988).

${ }^{10}$ J.-P. Puel and M. Yamamoto, C. R. Acad. Sci. Paris 320, série I, 1171 (1995).

${ }^{11}$ M. Yamamoto, Inverse Problems 11, 481 (1995).

${ }^{12}$ J.-L. Lions, C. R. Acad. Sci. Paris 302, série I, 471 (1986).

${ }^{13}$ J.-L. Lions, C. R. Acad. Sci. Paris 307, série I, 865 (1988).

${ }^{14}$ J.-L. Lions, SIAM Review 30, 1 (1988).

${ }^{15}$ D. L. Russell, SIAM J. Control and Opt. 4, 276 (1996).

${ }^{16}$ I. Lasiecka and R. Triggiani, Differential and Algebraic Riccati Equations with Applications to Boundary/Point Control Problems: Continuous Theory and Approximation Theory, in Lecture Notes in Control and Information Sciences, vol. 164 (Springer Verlag, New York, 1991).

${ }^{17}$ S. Lenhart, V. Protopopescu, and J. Yong, Applicable Analysis (to appear).

${ }^{18}$ M. Liang, preprint, University of Tennessee, 1997. 